\title{
PRESENTACIÓN
}

\section{EXPEDIENTE SOBRE LA CUESTIÓN DE LA ENSEÑANZA DE LA FILOSOFÍA}

Presentamos a continuación un breve recuento de las numerosas declaraciones y artículos aparecidos en la prensa, a propósito del anuncio, también dado a conocer en periódicos, de un proyecto que trata de la eliminación, entre otros, del ramo de filosofía en la enseñanza media y su fusión o reemplazo por una asignatura llamada "Educación Ciudadana". Solo incluimos en este expediente, por razones de espacio, el comunicado conjunto de la Facultad de Filosofía y Humanidades de la Universidad de Chile y el Senado de la misma institución, un editorial del diario El Mercurio, la declaración del Departamento de Filosofía de la Facultad de Filosofía de la Universidad de Chile, el artículo ¿"No más filosofía”? del Rector de la Universidad Diego Portales, el comunicado de la Reprofich y la llamada Declaración de Paris del Colegio Internacional de Filosofía, emitida con ocasión de un proyecto similar que se intentó implementar en Francia; la recibimos tan pronto se conoció la noticia afuera. Llama la atención que en ella se destaque en forma especial, la denominación: la enseñanza de la filosofía debe impartirse con ese nombre.

En vista de la pronta y masiva reacción que provocó este trascendido, las autoridades del Mineduc desmintieron que se tratara de suprimir la filosofía, sin contradecir, no obstante, el propósito de reducir la asignatura a una de carácter electivo. Esta rectificación -también incluida aquí- no zanjaba, pues, el diferendo. Por lo demás, no es la primera vez que surge una iniciativa análoga. Hace unos diez años, se intentó reducir el número de horas de la asignatura, de modo que la intención de minimizar la formación en filosofía está instalada, según toda evidencia, entre los expertos en currículos que asesoran a las autoridades. Y llama la atención que no hayan sido consultados para el efecto los propios filósofos, sino "especialistas" ajenos a las competencias, capacidades y disposiciones que desarrolla esta disciplina. No es extraño entonces, que se haya producido un diálogo de sordos, entre quienes aducían la necesidad de formar en la reflexión, el desarrollo del pensamiento libre y la conciencia crítica, y quienes invocaban urgencias de horario. De hecho, para un diálogo se requieren por lo menos dos, pero para un diálogo de sordos, basta uno solo que sea o se haga el sordo.

En el momento del cierre de esta edición, un grupo de profesores fue recibido en el Mineduc y se les prometió que la asignatura no sería eliminada; por el contrario, se mantendría en el plan básico. Pero, al mismo tiempo, se advertía que lo convenido en esa misma reunión no tenía carácter vinculante, pues cualquier acuerdo debía ser ratificado por el Consejo Nacional de Educación. Ese encuentro tendría el propósito 
de iniciar un diálogo y restablecer la confianza; lo cierto es que se había avanzado ya mucho en destruirla; desde luego, con el anuncio de eliminación y el error inicial de no haber consultado a tiempo a los implicados.

El Director 

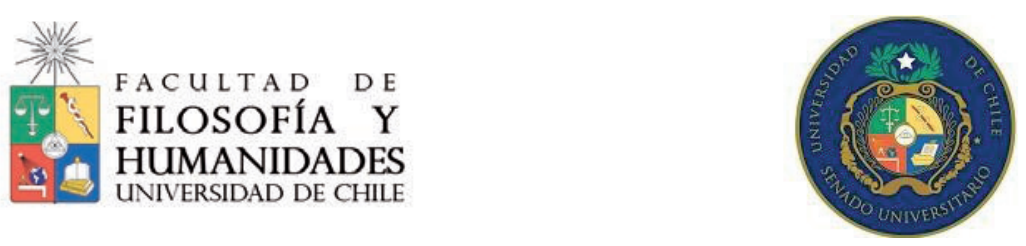

\section{Declaración Pública \\ Senado Universitario y Facultad de Filosofía y Humanidades Universidad de Chile}

Durante los años 2015 y 2016, una comisión transversal convocada por el Ministerio de Educación generó un conjunto de acuerdos y recomendaciones para el desarrollo de una Política Nacional de Desarrollo Curricular. Las acciones y decisiones de política pública que de ellas se están derivando no han sido discutidas y, de hecho, están poniendo en entredicho el principio que se invoca en todos los documentos, en el sentido de buscar una formación integral de las personas.

En días recientes, se ha difundido un documento del Ministerio de Educación titulado Reforma educacional en marcha, que incluye una "Propuesta de estructura curricular" para $3^{\circ}$ y $4^{\circ}$ año de Enseñanza Media, que introduce cambios sustantivos en el currículo escolar actual en dichos niveles. La propuesta distingue entre un Plan Común, que ahora se extiende a todas las modalidades de Educación Media en los niveles antes mencionados, así como un Plan Diferenciado.

De hecho, la propuesta de aprendizajes nucleares tal como está expresada en el documento de "Recomendaciones para una política Nacional de Desarrollo Curricular", no necesariamente permite desprender de ella, ni excluir como parte relevante de los saberes nucleares, a la Filosofía y la Historia, disciplinas fundamentales de la formación ciudadana e integral de las personas.

La estructura general del Plan Común incluye las siguientes áreas: "Artes", "Ciencia, Naturaleza y Sociedad", "Educación Ciudadana", "Educación Física y Salud", "Lengua y Literatura", "Matemática" y "Consejo de curso". En el Plan Diferenciado se incluyen las mismas áreas anteriores más "Filosofía", "Historia", "Geografía" "Ciencias sociales" "Inglés" y "Proyecto", de las cuales sólo estas últimas dos asignaturas serían obligatorias ${ }^{1}$.

Lo que se infiere de la propuesta es que la asignatura de Filosofía, con tres horas en $3^{\circ} \mathrm{y}$ tres en $4^{\circ}$ medio actualmente, ya no sería parte del Plan Común en Educación Media y que podría, en los hechos, estar ausente por completo de la formación de un estudiante

\footnotetext{
${ }^{1}$ Áreas entre las que se eligen cuatro en $3^{\circ}$ medio y dos en $4^{\circ}$ medio.
} 


\section{FACULTAD DE FILOSOFÍA Y HUMANIDADES UNIVERSIDAD DE CHILE}

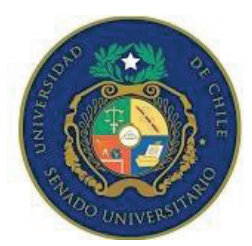

secundario chileno, si es que no la elige en el plan diferenciado. No obstante, en el Plan Diferenciado están también presentes asignaturas como Matemáticas y Lenguaje fundamentales para la rendición de la PSU-. Es muy probable que, por esta sola razón, sean éstas las asignaturas seleccionadas por los estudiantes.

En realidad, la situación que describimos es más grave aún, puesto que no es sólo Filosofía la que quedaría fuera del Plan Común, sino que también Historia, lo que significa que ámbitos fundamentales de las Humanidades podrían estar ausentes en la formación de muchos estudiantes de $3^{\circ}$ y $4^{\circ}$ medio.

La razón que se entrega en el documento para realizar dichos cambios, es que el currículo actual no incluye Filosofía e Historia, por ejemplo, en la modalidad Técnico Profesional ni en la modalidad Artística. Sin embargo, la propuesta realizada por el Mineduc no contempla generalizar estas disciplinas, sino transformarlas en electivas, lo que, en estricto rigor, no puede ser considerada como una solución adecuada frente a la inquietud manifestada.

Como Senado Universitario y Facultad de Filosofía y Humanidades, nos preocupan muy seriamente estas propuestas de cambio por varias razones:

- La primera es que la Enseñanza Media en Chile ha tenido, desde sus comienzos, un carácter de formación cultural general, que permite que los jóvenes comprendan y puedan evaluar críticamente el mundo en el que viven. Trata de "promover el desarrollo intelectual y moral de una nación“, decía Miguel Luis Amunátegui en 1897. La Educación Media no ha tenido ni tiene el propósito de introducir a una formación profesional ni especializada, dado que, en general, este tipo de formación es potestad de la Educación Superior. En este sentido, parece evidente que aquellas asignaturas que poseen claramente estos propósitos de formación humana general, como la Historia, las Ciencias Sociales y la Filosofía, deben incluirse como elementos básicos e indispensables, orientados a generar capacidad crítica en los integrantes de nuestra sociedad. La ausencia, o una presencia menor de estas disciplinas, como el propio Ministerio lo reconoce, sólo contribuye a aumentar la brecha de desigualdad que nuestro sistema escolar ya significa para los diferentes grupos en nuestra sociedad. 


\section{FACULTAD DE FILOSOFÍA Y HUMANIDADES UNIVERSIDAD DE CHILE}

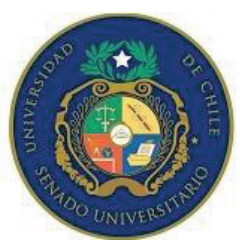

- En segundo lugar, el espíritu general de la modificación propuesta, más allá de la incorporación de la educación ciudadana -que, por lo demás, se adquiere y asimila mejor con actividades comunes y en la práctica-, margina el estudio de las Humanidades, para privilegiar, en todos los niveles del currículo, el estudio de disciplinas más en consonancia con las exigencias de pruebas internacionales estandarizadas, que surgen de organizaciones ligadas al fomento del comercio mundial y que nada tienen que ver con las necesidades y demandas de nuestros jóvenes. Con esto estamos dando, probablemente, un paso más hacia la "globalización" de nuestra enseñanza y su sujeción a demandas instrumentales que provienen del dominio productivo.

- Por último, esta propuesta surge en el contexto del desarrollo de intensas discusiones sobre la creación de un Ministerio de Ciencia y Tecnología, en las que pueden advertirse sesgos similares, que marginan a las Humanidades de la discusión sobre las formas y contenidos del saber y la cultura, que nuestra sociedad quiere y necesita fortalecer.

Es en este sentido que el Senado Universitario de la Universidad de Chile, junto a las autoridades de la Facultad de Filosofía y Humanidades, expresan su profunda preocupación y rechazo de lo que parece un nuevo paso hacia la marginación de las humanidades -y también de las ciencias, en sus aspectos no instrumentales- de nuestra cultura común.

Esperamos, como Senado Universitario y como Facultad de Filosofía y Humanidades de la Universidad de Chile, junto a las organizaciones y personalidades de la cultura chilena que ya han expresado un rechazo similar, que esta medida se revierta y se proceda a una revisión de nuestro currículo secundario tomando en cuenta todas estas opiniones y orientaciones que apuntan a repensar nuestro currículo con un sentido más profundo y sin visiones estereotipadas. 


\section{Reforma curricular extraviada}

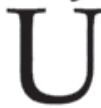

na fuerte reacción adversa ha despertado la propuesta de la unidad técnica pertinente del Ministerio de Educación para reformar la estructura curricular del plan común de la educación científicohumanista y técnica. Especial rechazo causa la desaparición de la historia y la filosofía como disciplinas autónomas. Respecto de esta última, que se impartía en tercero y cuarto medio, explico la autoridad que sus contenidos se incluirían en la nueva asignatura - propuesta por la comisión Engel- llamada "Educación ciudadana", sin perjuicio de poder impartirse también como asignatura electiva, lo cual, de ser verdadero lo primero, es inconsistente con lo segundo: ¿para qué ofrecer como electivos los mismos contenidos que ya estarían cubiertos por la nueva asignatura?

La reforma parece fundarse en errores conceptuales graves. La filosofía, ante todo, excede la formación ciudadana, a la cual sin duda contribuye e incluso, para influyentes pensadores, es maestra de civilidad. La filosofía, desde sus orígenes, ha mostrado una "devoción" — como lo definió un pensador- por la búsqueda y el preguntar. Por cierto que la historia de la filosofía contiene una secuencia de respuestas tentativas, pero su rasgo distintivo respecto de cualquier otra disciplina es su obsesión por dar con las preguntas correctas, aquellas preguntas que los demás saberes no se formulan o lo hacen mal, preguntas que se refieren a opiniones aceptadas como presupuestos incuestionables, de suyo evidentes

La filosofía, incluso en sus áreas más especulativas, es un ejercicio sistemático por cuestionar lo que en apariencia no requiere ser cuestionado. Un ejemplo claro y urgente se refiere al preguntarse por los límites y fundamentos de la ciencia contemporánea o por la común naturaleza del ser humano. La amputación de la filosofía del currículum genera, así, una carencia grave en la formación de los alumnos, desvinculándolos de una dimensión esencial de nuestra tradición cultural. No se trata de plantear a la filosofía como panacea y, desde luego, sería importante someter a una profunda revisión los programas, textos de estudio y metodología de su enseñanza, pero su eliminación parece una mala serial - cuyo origen no puede sino atribuirse a una tosca desvalorización- para las nuevas generaciones y golpe muy duro para el cultivo de las humanidades en nuestro país. La absorción de Historia y Ciencias Sociales dentro de un ámbito disciplinario inédito ("Ciencia, naturaleza y sociedad") no puede sino dar lugar a inquietudes análogas.

En la reforma propuesta se advierte una tendencia titubeante a borrar los límites disciplinarios; titubeante, porque solo alcanza a algunas asignaturas y a otras no. Matemáticas, que se mantiene como autónoma, ¿acaso no es una ciencia? Lo mismo puede ser preguntado respecto de "arte", que bien podría entenderse como parte de "sociedad". La reforma, por otro lado, parece ampliar el campo de la literatura, al introducir el ramo de "Lenguaje y literatura", lo cual es un progreso para las humanidades, pero, por otro lado, hace desaparecer la filosofía y la historia como disciplinas independientes. Cabe preguntarse, pues, ¿cuál es el rumbo de esta propuesta? Urge, por lo mismo, una discusión pausada, abierta a la sociedad y con la mira puesta en los intereses y formación de los alumnos. 
FACULTAD DE

FILOSOFÍA Y HUMANIDADES

UNIVERSIDAD DE CHILE

\section{Por el derecho a la filosofía en la educación media}

De un modo sorpresivo y azaroso, sin consulta previa a este Departamento de Filosofía ni a ningún otro, nos ha llegado la información según la cual la asignatura de Filosofía desaparecería del Plan Común para Tercero y Cuarto Medio. La información entregada por Mineduc hasta el momento, indica que la asignatura pasa a ser optativa, ofreciéndose a cambio una eventual y poco clara participación de los profesores de Filosofía en la nueva asignatura de "Educación ciudadana". Nos parece que la propuesta conocida del Mineduc es muy desalentadora.

Resulta inquietante $\mathrm{o}$ al menos preocupante la eliminación de la asignatura de Filosofía, pues no son pocas ni menores las consecuencias de una decisión que no cuenta hasta ahora con un texto
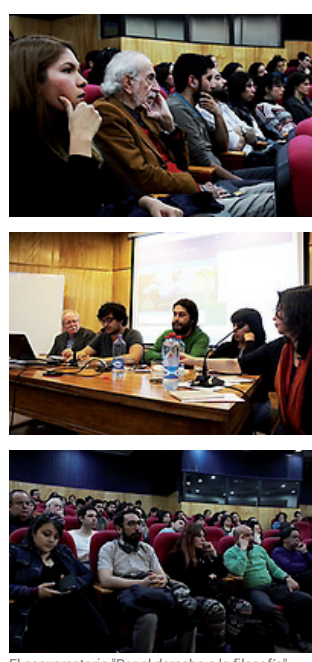

El conversatorio "Por el derecho a la filosofía" congregó a la comunidad en torno a las lecturas
posibles respecto de la propuesta del Mineduc. que dé razones o justificaciones valederas. Esta decisión supone, por de pronto, una muy drástica reducción de un saber que ha acompañado a la humanidad desde muy antiguo, que ha jugado un papel fundamental en la propia constitución de las repúblicas en América Latina y Chile, que no es disociable del desarrollo y de las rupturas que se han dado en otras disciplinas y artes, ni tampoco del despliegue - ciertamente no exclusivo de la filosofía - de una serie de habilidades reflexivas, argumentativas y críticas necesarias para la 
formación integral del educando y para una comprensión y problematización de un presente múltiple y complejo; necesarias igualmente para la formación de un ciudadano cuya independencia o capacidad de juicio propio son indispensables para el fortalecimiento y ampliación de la democracia.

La decisión del Mineduc, en caso de implicar efectivamente la eliminación del ramo de Filosofía, precariza la formación del (de la) alumno (a), lo cercena de fuentes espirituales y reflexivas que son constituyentes de la experiencia humana, de autores, temas o discusiones que han marcado la historia de la humanidad, de preguntas o dilemas existenciales o éticos que se ha planteado o se plantea hoy la filosofía. Lo cercena también de la comprensión de bases culturales y valóricas distintas a las propias, de otras matrices o modos de pensar y de vivir. La propuesta del Mineduc representaría así un atentado a la tolerancia y a la diversidad, a la constitución del educando como sujeto de discurso, y a su derecho de imaginar mundos distintos a aquellos no declarados, pero sí presentidos, por una decisión que con dificultad oculta su carácter instrumental o de rígido control o medición de los efectos educativos.

Si a esto se suma, según se ha venido informando, la eliminación de Historia como asignatura obligatoria del Plan Común para Tercero y Cuarto Medio - información que ha aparecido en conjunto con la referida a la filosofía y bajo los mismos términos - podemos presumir una muy preocupante ofensiva contra las humanidades. Esta ofensiva impediría que los estudiantes de la fase terminal del ciclo escolar puedan hacerse cargo crítica o activamente de algunos de los más importantes problemas de la vida y de la historia de los hombres y de las mujeres de hoy, a través de su experiencia como jóvenes, del desarrollo de su propia convivencia, y por medio de argumentaciones rigurosas y conceptos precisos.

Lo que está en juego no es pues poca cosa: la calidad y el sentido de la educación de los jóvenes, el fortalecimiento de una educación pública que no puede ser ajena a la pregunta por el país y la modernidad que se busca, así como la necesidad de generar un amplio diálogo, con todos los actores involucrados, respecto del "para qué" o el horizonte que guía la reforma en la educación. 
Hacemos nuestra la Declaración del Senado Universitario y la Facultad de Filosofía y Humanidades de la Universidad de Chile que, junto con señalar la necesidad de repensar los programas actuales de Filosofía en la Educación Media, exige además la inmediata revocación de una propuesta inconsulta, que negaría a nuestros jóvenes no solo su legítimo derecho a la filosofía en sus variadas formas y temas, sino también, o junto con ello, su capacidad de analizar y discutir racional, informada y libremente los problemas del mundo.

Departamento de Filosofía, Universidad de Chile

Miércoles 7 de septiembre de 2016 


\section{¿No más filosofía?}

Carlos Peña

Se ha propuesto una reforma para la estructura curricular de tercero y cuarto medio que suprime del currículum obligatorio la enseñanza de la filosofía. De aprobarse, sería, de allí en adelante, un ramo simplemente electivo.

Los prejuicios contra la filosofía, como si ella fuera un quehacer prescindible, un entretenimiento carente de toda utilidad, un artificio conceptual que aleja de las cosas urgentes e importantes de la vida, es muy viejo según lo prueba una fábula de Esopo que recogió Platón en el diálogo Teeteto. "Se cuenta de Tales --se lee en Teeteto, 174 AB-- que mientras se ocupaba de la bóveda celeste, mirando hacia arriba, cayó en un pozo. Se rió de él entonces una sirvienta tracia, jocosa y bonita, diciéndole que mientras deseaba con toda pasión llegar a conocer las cosas del cielo, le quedaba oculto aquello que estaba ante su nariz y bajo sus pies. La misma burla vale para todos aquellos que se introducen en la filosofía", añade Platón.

Ese prejuicio antifilosófico, cuando se lo deja crecer hasta el extremo que logre espantar a la filosofía del ciclo final de la trayectoria escolar, es perjudicial para la cultura.

Porque ocurre que la filosofía, al revés de lo que imaginaba la muchacha tracia del cuento, no se ocupa de las cosas del cielo, sino de la condición humana, de las circunstancias que aquejan y desasosiegan a una época. Cuando Kant se esforzó porque la metafísica tomara el camino seguro de la ciencia, estaba en verdad intentando salvar, mediante la razón, a cosas como el alma, Dios o la inmortalidad, que subyacían a la cultura pública de su tiempo. Era, pues, esta última la que le interesaba. Y cuando Hegel escribió su filosofía del derecho, no se estaba ocupando del "cielo de los conceptos" (Ihering) sino de reconciliar a los seres humanos con las instituciones de su época, mostrándoles el potencial normativo que las inspiraban. Y en fin, cuando Rawls escribió su Teoría de la Justicia no lo hizo para distraer a sus lectores o desorientar a los economistas, sino para contribuir a que la política de una sociedad moderna y democrática fuera más reflexiva, más consciente de sus posibilidades y sus límites. Por eso Heidegger tiene toda la razón cuando observa que si con la filosofía no se puede hacer nada, quizá ella pueda hacer algo con nosotros.

¿Y qué puede hacer con nosotros la enseñanza de la filosofía? 
Ante todo, la filosofía ayuda a aguzar la capacidad reflexiva y crítica de las personas. En su afán por preguntarlo todo, llevando el razonamiento hasta el límite de sus posibilidades (así definía la filosofía Jorge Millas) la filosofía puede ser un antídoto eficaz contra el prejuicio. Al demandar el fundamento de todo lo que se pone delante suyo, la filosofía puede enseñar, a quienes se asoman a ella, a rechazar las ideas recibidas e incitar a las personas a pensar por sí mismas, a pasar, según la famosa fórmula kantiana, de ser un pupilo a ser un adulto intelectualmente hablando.

En la medida que la filosofía ayuda a las personas a pensar por si mismas y a espantar el prejuicio, ayuda, por supuesto, a que la vida cívica sea también más reflexiva y tolerante y a que el ideal de una democracia donde sus miembros deliberen y razonen, sea un ideal al que, siquiera en algunos momentos, nos podamos acercar.

Pero, ante todo, la filosofía ayuda a evitar que una forma específica del prejuicio -lo que pudiera llamarse el prejuicio técnico-- se enseñoree del espacio público y de las decisiones que atingen a todos.

El prejuicio técnico -una de cuyas víctimas predilectas es la propia filosofía-- da por resueltas cosas que deben ser pensadas. Por eso Heidegger dijo que la ciencia no piensa. El prejuicio técnico enseña que la vida social, la forma en que se organizan las instituciones y el modo en que concebimos la vida social, es el fruto de una simple racionalidad instrumental, el resultado ante todo de la búsqueda de eficiencia y de funcionalidad. Al plantear así las cosas, ese tipo de racionalidad oscurece las cuestiones más sustantivas que subyacen en la cultura y que una democracia se esfuerza por entregar a la deliberación de los ciudadanos. Esas cuestiones sustantivas asoman cuando se debate sobre el aborto, las pensiones o la constitución, y ninguna de ellas, por más que sus cultores se esmeren, puede ser resuelta ajustando medios a fines que nunca se explicitan ni se discuten.

No es, pues, la suerte de la filosofía y de sus cultores la que se juega en una decisión como la de suprimir la enseñanza de la filosofía del currículum escolar, es la fisonomía de la cultura pública en Chile la que, siquiera en parte, se arriesga en esa decisión. Uno de los rasgos de una cultura moderna es la autonomía que enseña a sus miembros; pero una autonomía que no está acompañada de capacidad reflexiva, de la insolencia de preguntar hasta el límite -todas cosas que la enseñanza de la filosofía puede ayudar a adquirir-- es una autonomía meramente formal, es una autonomía indefensa frente al prejuicio y al poder.

(Publicado en el diario El Mercurio de Santiago de Chile, el 25 de agosto de 2016) 
FACULTAD DE

FILOSOFÍA Y HUMANIDADES

UNIVERSIDAD DE CHILE

\section{Comunicado de la Red de Profesores de Filosofía de Chile REPROFICH}

La REPROFICH expresa su total rechazo a la "Propuesta de estructura curricular $3^{\circ}$ y $4^{\circ}$ medio" divulgada por representantes de la Unidad de Currículum y Evaluación del Ministerio de Educación en liceos y filtrada a distintas universidades del país donde la Filosofía deja de estar en el plan común, para ser relegado al campo de una especialidad electiva.

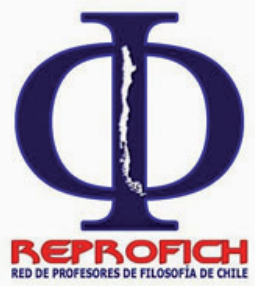

Este documento en formato Power Point presenta la nueva estructura curricular de la Enseñanza Media que, no tan solo prescinde de la Filosofía en el Plan Común General, sino que también las asignaturas de Historia, Física, Química y Biología quedan fuera de esta base donde el Mineduc pretende establecer una estructura curricular que cumpla con una educación igualitaria para los planes diferenciados Técnico Profesional, Científico Humanista y Artístico. De este modo, la enseñanza de la Filosofía queda relegada a la Estructura Común del Plan Diferenciado como Especialización en Filosofía lo que implicaría tres electivos de Extensión en Filosofía, definición que no deja clara la propuesta. Desde esta perspectiva, ¿qué tipo de educación está pensando y proponiendo el Mineduc con esta propuesta que discrimina asignaturas sin el debido diálogo y debate con las distintas organizaciones de profesores? ¿Ha sido el Colegio de Profesores una institución representativa de las demandas de las y los profesores en la 
mesa de desarrollo curricular que presentó el documento "Recomendaciones para una política nacional de desarrollo curricular"?

Es bajo este contexto que la Reprofich considera que la eliminación de la enseñanza de la filosofía del Plan Común General de $3^{\circ}$ y $4^{\circ}$ Medio:

Atenta contra la educación pública y de "calidad" que el actual gobierno se comprometió a defender y a desarrollar a través de la Reforma Educacional que hoy está en marcha. En efecto, no considerar la filosofía como una asignatura base de los estudiantes chilenos, es rechazar la posibilidad de asegurar un espacio institucional educativo en el que se adquieran conocimientos, aprendizajes y habilidades que impliquen el desarrollo del pensamiento filosófico como condición fundamental de la formación integral de ciudadanos libres.

Carece de fundamentos filosóficos de la educación centrándose en valores y principios técnicos que revelan una inconsistencia en la eliminación de la enseñanza de la filosofía, a saber:

en este documento se propone como una de las tres habilidades principales del Plan Común General el Pensamiento Crítico. En este sentido, queda de manifiesto la evidente inconsistencia conceptual y teórica de lo que se entiende como pensamiento crítico, y la ignorancia respecto a las nuevas propuestas didácticas de la enseñanza de la filosofía en las que se promueve el pensar y el reflexionar de manera colaborativa y comunitaria donde las y los estudiantes no solo conocen el pensamiento de distintos autores de la historia de la disciplina, sino que crean y desarrollan ideas propias nutriéndose de las ideas de los otros a través del diálogo y de la reflexión colectiva.

en este documento se propone una base cultural del plan común que orienta "la formación de los y las estudiantes como personas integrales y ciudadanos conscientes y responsables de su entorno social, cultural y natural". Objetivo que consideramos que es propio -no por ello exclusividad- de la enseñanza de la filosofía a través de los problemas y las preguntas que fundamentan la enseñanza de la ética, de la filosofía política, de la filosofía de las ciencias, de la filosofía de la naturaleza, etc. el Plan Común es concebido por el documento ministerial como "un espacio de síntesis, reflexión e integración de los aprendizajes desarrollados hasta segundo medio". Bajo esta perspectiva afirmamos 
que la filosofía es una disciplina y una práctica que permite dar una visión integradora, holística y de sentido a esta base común en la medida en que el ejercicio de la razón permite el análisis, síntesis e integración de distintos aprendizajes recibidos en la escolaridad como en la vida cotidiana. Por tanto, suprimirla de la formación general es un contrasentido.

esta decisión no contempló la experiencia, el saber y los conocimientos de las y los profesores de filosofía aun cuando la Reprofich se reunió en dos ocasiones con los encargados de educación y de currículum del Ministerio solicitando formar parte del trabajo de la reforma al currículum. Además, se solicitó información a través de cartas a la ministra Del Piano las que nunca fueron respondidas. Junto a este accionar, la propuesta aquí en cuestión no considera recomendaciones que a nuestro juicio son fundamentales en la construcción de un nuevo currículum para la educación pública chilena. En efecto, el "Informe de la mesa de desarrollo curricular" presentado por la Unidad de Currículum y Evaluación del Ministerio de Educación a través del documento "Recomendaciones para una política nacional de desarrollo curricular" de mayo de 2016, en el apartado "A" titulado "Producción curricular" recomienda: "Resguardar que la gestación del currículum nacional sea participativa y transparente desde la perspectiva de la ciudadanía: el currículum debe recoger necesidades y demandas en su proceso de creación, articulando las expectativas de diferentes actores y grupos sociales, lo que permite que se convierta en una definición curricular que refleje lo más cercanamente posible esas expectativas y que combine criterios de rigor técnico y legitimidad social".

La "Propuesta de estructura curricular $3^{\circ}$ y $4^{\circ}$ medio" es una evidencia de la inconsistencia, desarmonización y desorganización de la actual reforma a la educación. En efecto, la eliminación de las horas de Filosofía en el plan común no se condice con el desarrollo de la carrera de Pedagogía en Filosofía en las distintas universidades del $\mathrm{CRUCH}$ materializado en los procesos de acreditación y de innovación curricular, no contemplando una visión global de educación ni tampoco resguardando los recursos económicos, laborales y de conocimiento invertidos en estos procesos. 
A partir de los argumentos recién expuestos la Reprofich llama al Ministerio de Educación a:

reincorporar la filosofía en el plan común y mantenerla en un plan electivo asegurando su enseñanza en las tres modalidades que el currículum contempla, a saber, en la formación Técnico Profesional, Científico Humanista y Artística.

extender la enseñanza de la filosofía para $1^{\circ}$ y $2^{\circ}$ medio. mantener y potenciar la enseñanza de la filosofía en la Educación de Adultos.

desarrollar un nuevo Programa de Estudios de la asignatura de Filosofía para la Educación Media en conjunto con las y los profesores asociados a la Reprofich, con académicos y con estudiantes de Pedagogía en Filosofía. Esto en función de renovar y actualizar los Planes y Programas vigentes los cuales no han sido revisados desde su implementación en la última reforma curricular en el año 2001.

En consecuencia, la Reprofich llama a todas y todos los profesores de filosofía de Chile, profesores en general, movimientos de profesores, Colegio de Profesores, movimientos ciudadanos, fundaciones de investigación, movimientos y partidos políticos y a la ciudadanía en general a DEFENDER EL DERECHO A LA FILOSOFÍA EN LA EDUCACIÓN PÚBLICA CHILENA.

\section{Coordinación Nacional} REPROFICH 


\section{DECLARACIÓN DE PARÍS EN FAVOR DE LA FILOSOFÍA}

Nosotros, los participantes en las Jornadas internacionales de estudio sobre "Filosofía y democracia en el mundo" organizadas por la UNESCO en París los días 15 y 16 de febrero de 1995, señalamos que los problemas de que la filosofía se ocupa son los problemas universales de la vida y la existencia humanas; creemos que la reflexión filosófica puede y debe contribuir a la comprensión y la orientación del quehacer humano; consideramos que la práctica de la filosofía, que no excluye ninguna idea del libre debate y se esfuerza por establecer definiciones exactas de los conceptos utilizados a fin de comprobar la validez de los propios razonamientos y efectuar un examen riguroso de los ajenos, permite a todas las personas aprender a pensar con independencia; hacemos hincapié en que la enseñanza de la filosofía estimula la apertura mental, la responsabilidad civil, el entendimiento y la tolerancia entre las personas y los grupos; insistimos en que la educación filosófica, al inducir a la independencia de criterio, la reflexión y la resistencia a las diversas formas de propaganda, prepara a todas las personas a asumir sus responsabilidades ante las grandes cuestiones del mundo contemporáneo, especialmente en el plano ético; confirmamos que el fomento del debate filosófico en la educación y la vida cultural constituye una aportación primordial a la formación de los ciudadanos al poner en ejercicio su capacidad de juicio, que es fundamental en toda democracia. Así pues, comprometiéndonos a hacer todo lo que podamos en nuestras instituciones y en nuestros países respectivos para lograr estos objetivos, declaramos lo siguiente: Todo individuo debe tener derecho a dedicarse al libre estudio de la filosofía bajo cualquier forma y en cualquier lugar del mundo; la enseñanza de la filosofía debe mantenerse o ampliarse donde ya existe, implantarse donde aún no existe y ser nombrada explícitamente con la palabra "filosofía"; la enseñanza de la filosofía debería ser impartida por profesores cualificados e instruidos específicamente a tal efecto y no estar supeditada a consideración económica, técnica, religiosa, política o ideológica alguna; aun manteniendo su autonomía, la enseñanza de la filosofía debería vincularse en la medida de lo posible a la formación académica o profesional en todos los campos. A fin de impartir educación filosófica a adultos se fomentará la distribución de libros accesibles por la lengua en que estén editados y por su precio de venta a un amplio público lector, la producción de programas de radio y televisión y de cintas audio 
y vídeo, el uso con fines educativos de todo tipo de tecnología audiovisual e informativa, el fomento de múltiples situaciones en las que se celebren libremente debates y todo tipo de iniciativas encaminadas a proporcionar al mayor número de personas posible conocimientos básicos sobre cuestiones y métodos filosóficos. Las instituciones de investigación y enseñanza deberían perseguir y respaldar el conocimiento de los planteamientos filosóficos de las distintas culturas, la comparación de lo que cada una puede aportar y el análisis de lo que las acerca y lo que las separa; la filosofía, en tanto que libre actividad de indagación, no puede establecer juicios definitivos y fomenta el respeto de las convicciones individuales, pero, en ningún caso, a riesgo de negar su propia naturaleza, deberá aceptar doctrinas que denieguen la libertad ajena, afrenten la dignidad humana o siembren la barbarie. 


\section{DECLARACIÓN PÚBLICA}

En relación a declaraciones que han circulado sobre la asignatura de Filosofía, queremos señalar de manera enfática que no se eliminará la asignatura de Filosofía da la formación general del currículum de $3^{\circ}$ y $4^{\circ}$ año medio. Muy por el contrario, en el Ministerio de Educación creemos que la formación ética, el espíritu crítico y el aporte de las humanidades son fundamentales en la formación de niñas, niños y jóvenes. Por este motivo estamos estudiando de qué manera pueden incorporarse estas disciplinas también en el currículum de los estudiantes de establecimientos de formación Técnico Profesional y en los liceos artísticos.

El desarrollo de Bases Curriculares para $3^{\circ}$ y $4^{\circ}$ medio es un mandato de la Ley General de Educación, dado que son los únicos niveles que no cuentan con definición curricular conforme a esta ley.

Reiteramos también que el Ministerio de Educación no tiene la facultad de realizar ajustes curriculares por sí solo. Las propuestas del Ministerio deben ser presentadas al Consejo Nacional de Educación, un ente autónomo, para su evaluación y aprobación.

Adriana Delpiano Puelma

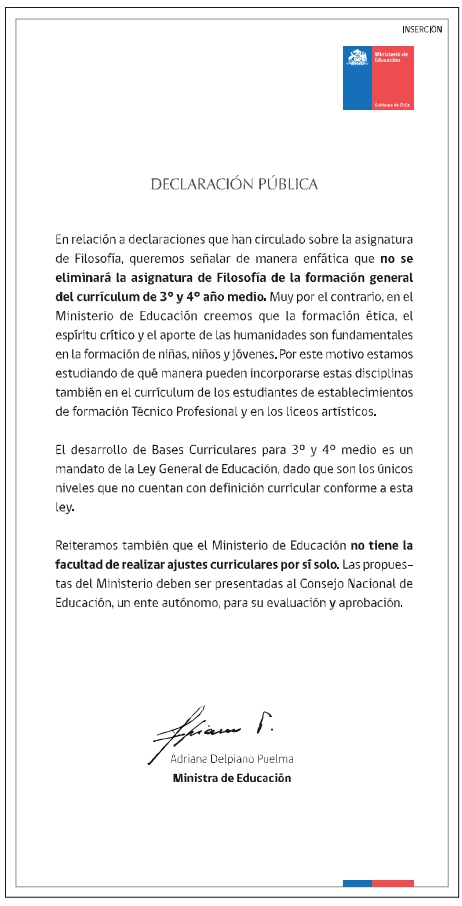

Ministra de Educación 